\title{
TAGUNG
}

\section{Der Weg nach vorn führt zurück: Zur geschichtlichen Dimension des europäischen Konstitutionalisierungsprozesses}

\author{
Sebastian Wolf*
}

Es ist keine leichte Aufgabe, die Ergebnisse einer Tagung zu rekonstruieren, auf der vielfach von Dekonstruktion die Rede war. Bereits die Ausgangsthese der Veranstaltung, die Geschichte lasse sich im Prozess der Konstitutionalisierung Europas nicht verdrängen und dürfe deshalb nicht künstlich unterdrückt werden, ist als eine Kritik an einer vor allem in Hochglanzbroschüren und Festtagsreden beschworenen, reduktionistischen Sicht der europäischen Integrationsgeschichte zu verstehen. Nach dieser ,Meistererzählung' entschieden sich kurz nach dem Zweiten Weltkrieg einige weitsichtige Staatsmänner für ein visionäres supranationales Projekt, das die europäischen Völker seitdem immer enger zusammenschweißt und ihnen unablässig eine Fülle von Wohltaten beschert (weshalb sich ernsthafte Kritik an seiner Fortentwicklung durch die heutigen politischen Eliten tunlichst verbietet). Der Europäische Konvent hätte eine solche allzu euphorische Geschichtsdarstellung gepaart mit einem naiven Fortschrittsglauben gerne in der Präambel des Verfassungsvertrags verankert; es ist wohl einer Initiative Polens zu verdanken, dass die Regierungskonferenz die Wörter ,schmerzliche Erfahrungen“ einfügte als knappe Reminiszenz an all die historischen Feindschaften zwischen den europäischen Völkern, die in länderübergreifender totalitärer Gewaltherrschaft, ideologischer Gleichschaltung, Völkermord und Weltkrieg gipfelten und als zentrale Hintergrundfolie für die Schaffung der Europäischen Gemeinschaften zu sehen sind.

Das Integrationsprojekt sieht sich trotz seiner unumstrittenen Erfolge auch über 60 Jahre

\section{„Bittere Erfahrungen“" der Vergangenheit und der Prozess der Konstitutionalisierung Europas}

Wissenschaftliche Tagung des Arbeitskreises Europäische Integration (AEI) in Zusammenarbeit mit dem Europäischen Hochschulinstitut Florenz, der Freien Universität Berlin und der Hertie School of Governance, Berlin Berlin, 20./21. Oktober 2006

\section{Wissenschaftliche Leitung:}

Prof. Dr. Christian JOERGES, Europäisches Hochschulinstitut Florenz

PD Dr. Matthias MAHLMANN, Freie Universität Berlin

Prof. Dr. Ulrich K. PREUSS, Hertie School of Governance, Berlin

Zur Geschichtlichkeit der Integration - Geschichtliche Rekonstruktionen und rechtliche Konstruktionen

Moderation:

Prof. Dr. Ulrich K. PREUSS

Die Gegenwart der Vergangenheit in Europa: das Beispiel der Deutsch-Polnischen Beziehungen

Prof. Dr. Gesine SCHWAN, Europa-Universität Franfurt/Oder

Historische Divergenzen als Problem einer Europäischen Zeitgeschichtsschreibung

Prof. Dr. Konrad H. JARAUSCH, University of North Carolina, Chapel Hill

Kommentare:

Prof. Dr. Mattias KUMM, New York University School of Law, New York

Prof. Dr. Jerzy KRANZ, Warsaw School of Economics, Warschau

Aufgaben und Streitpunkte einer Konstitutionalisierung Europas

Moderation:

PD Dr. Matthias MAHLMANN

Prof. Dr. Christian TOMUSCHAT, HumboldtUniversität zu Berlin

* Dr. Sebastian Wolf, LL.M.Eur., Sektionsreferent der Sektion I, Deutsches Forschungsinstitut für öffentliche Verwaltung, Speyer. 
nach Ende des Zweiten Weltkriegs und mehr als 15 Jahre nach dem Fall des Eisernern Vorhangs immer noch mit den langen Schatten der Vergangenheit konfrontiert. Tradiertes, oftmals unterschwelliges Geschichtsbewusstsein geht sowohl in die Handlungen derer ein, die direkt an der Konstitutionalisierung Europas mitwirken, als auch jener, die als Adressaten die Unionsverfassung interpretieren. Diesem Themenspektrum jenseits des Mainstreams von Europapolitik und Integrationsforschung war die Tagung gewidmet, über die an dieser Stelle zu berichten ist. Im Folgenden sollen in der Form eines überblicksartigen Essays zentrale Gegenstände und ausgewählte Grundfragen der Tagung rekonstruiert werden.

\section{Die Einbeziehung von Geschichte als Chance und Gefahr}

In den ersten Jahrzehnten des Integrationsprozesses war die bellizistische Vergangenheit Europas als Entstehungshintergrund der Europäischen Gemeinschaften bei den politischen Eliten noch sehr präsent. Aus der europäischen Geschichte voller ,schmerzlicher Erfahrungen" glaubte man nicht zuletzt Legitimation für das neue supranationale Projekt ableiten zu können. Dieses Geschichtsbewusstsein verblasst offenbar zunehmend, wie das oben genannte Beispiel des Europäischen Konvents anschaulich belegt. Bei der Diskussion um die künftige Konstruktion Europas spielen historische Argumente jedoch weiterhin eine nicht zu unterschätzende Rolle.

In letzter Zeit wird die europäische Geschichte vor allem von jenen zur Sprache gebracht, die etwa Angst vor einer zu mächtigen oder zu großen Europäischen Union haben. So wird beispielsweise argumentiert, der historisch gewachsene Nationalstaat mit seinen zahllosen Errungenschaften dürfe im Integrationsprozess nicht noch weiter ausbluten, oder die Türkei könne bereits aufgrund ihrer Vergangenheit nicht Teil der Union als eines Produkts der westlichen Zivilisationsgeschichte werden. Diejenigen, die Geschichte in dieser Form selektiv instrumentalisieren, scheinen in
Ist eine europäische Identität möglich? Oder: Wie wir lernen sollten, Zwiebeln zu lieben

PD Dr. Johannes POLLAK, Österreichische Akademie der Wissenschaften, Wien

Kommentar:

Dr. Felix HANSCHMANN, Universität Frankfurt/Main

Unbezähmbare Öffentlichkeit - Europa zwischen transnationaler Klassenherrschaft und egalitärer Konstitutionalisierung

Prof. Dr. Hauke BRUNKHORST, Universität Flensburg

Kommentar:

Alexandra KEMMERER, Universität Würzburg

Das Politische und das Soziale: eine unzureichende europäische Relation in historischer Perspektive Prof. Dr. Bo STRÅTH, Europäisches Hochschulinstitut Florenz

Kommentar:

Prof. Dr. Alexander SOMEK, University of Iowa, Iowa City

Unitas in Pluralitate als Verfassungsauftrag: Plädoyer für einen ,horizontalen Konstitutionalismus “ Prof. Dr. Christian JOERGES

Kommentar:

Dr. Fabrice LARAT, Universität Mannheim

Die Menschenrechtskonzeption Europas PD Dr. Matthias MAHLMANN

Kommentar:

Dr. Tobias HERBST, Humboldt-Universität zu Berlin

Der Europäische Föderalismus-Streit: Eine Unionsverfassung jenseits von Bundesstaat und Staatenbund?

Prof. Dr. Stefan OETER, Universität Hamburg

Kommentare:

Prof. Dr. Daniel HALBERSTAM, University of Michigan, Ann Arbor

Prof. Dr. Christoph MÖLLERS, Universität Göttingen

\section{Der Umgang mit Geschichte}

Moderation:

Prof. Dr. Christian JOERGES, Europäisches Hochschulinstitut Florenz

Thomas Manns Albtraum? Potenzial und Paradoxien europäischer Erinnerungspolitik

Prof. Dr. Jan-Werner MÜLLER, Princeton University

Kommentar:

Helmut KUHNE, MdEP, Brüssel/Soest

Aufarbeitung der Vergangenheit durch Recht? Prof. Dr. Hubert ROTTLEUTHNER, Freie Universität Berlin

Kommentar:

Prof. Dr. Peter NIESEN, Technische Universität Darmstadt

Kann Europa aus der Aufarbeitung seiner Vergangenheit eine neue Legitimität gewinnen?

PD Dr. Michael JEISMANN, Frankfurter Allgemeine Zeitung, Frankfurt/Main

Prof. Dr. Ulrich K. PREUSS 
den europapolitischen Diskursen mittlerweile wesentlich stärker vertreten zu sein als jene, die aus der Vergangenheit Argumente für verstärkte Vergemeinschaftung beziehungsweise alternative Integrationsmodelle schöpfen oder auch nur den Status Quo verteidigen.

Angesichts der skizzierten Gefahr der Instrumentalisierung von Geschichte zur Unterfütterung integrationsfeindlicher Politiken stellt sich die Frage, ob man historische Argumentationsstränge nicht von vorneherein aus der Debatte über die zukünftige Gestalt der Union ausschließen sollte. So lässt sich etwa darlegen, die Europäische Kommission sei für die Beurteilung der Beitrittsreife eines Landes wesentlich besser geeignet als populistisch agierende Politiker oder konservative Historiker, da sie in scheinbar objektiv technokratischer Manier lediglich nach rechtlichen und ökonomischen Kriterien bewerte. Die mit dieser Ansicht verbundene bewusste Ausblendung der geschichtlichen Dimension in guter Absicht erscheint dennoch unbefriedigend. Zum einen wird damit Konfliktpotenzial nicht beseitigt, sondern nur notdürftig verdeckt, und zum anderen beraubt man sich so schon im Vorfeld der Möglichkeit, aus der Aufarbeitung der europäischen Vergangenheit und ihrer Einarbeitung in die Verfassungsstruktur der Union vielleicht neue Legitimität zu gewinnen. Hierfür bedarf es allerdings wohl eines „gemeinsamen historischen Besitzstands", der bis jetzt höchstens in Ansätzen existiert.

\section{Hin zu einem acquis historique?}

Die Beschäftigung mit den dunklen Seiten der europäischen Vergangenheit ist geprägt durch Ungleichzeitigkeiten und nationale Verengungen. Verschiedene Akteure begannen in jedem Land zu jeweils unterschiedlichen Zeitpunkten mit der Aufarbeitung nationalsozialistischer, faschistischer oder kommunistischer Perioden. Die europäische Geschichte wird hier nicht selten auf eine nationale Selbstdarstellung verengt, in der symbolische Heldentaten einerseits und Opferstilisierung andererseits eine wichtige Rolle spielen. Wie könnte vor diesem komplexen Hintergrund ein acquis historique als Grundlage einer geschichtsbewussten Konstitutionalisierung Europas aussehen?

Eine quasi-vergemeinschaftete europäische Geschichtsschreibung mit zentralen Deutungseliten in Brüssel oder Straßburg erscheint wenig sinnvoll. Die Mitgliedstaaten und ihre Bürger würden es - wohl zurecht kaum hinnehmen, wenn ihre vertrauten Geschichtsdarstellungen plötzlich zentral umkodiert würden. Das Projekt eines acquis historique ist wohl eher als ein prozedurales Experiment zu verstehen, als eine wechselseitige Öffnung der nationalen Geschichtsschreibungen in Europa. Eine Grundvoraussetzung hierfür wäre nicht nur die bewusste Entscheidung zur Beschäftigung mit dem jeweiligen Vorverständnis des Anderen, sondern auch die Bereitschaft zur selbstreflexiven Auseinandersetzung mit der eigenen selektiven, national verengten Geschichtsinterpretation.

Diese Perspektive lässt den im Beitrittsprozess umstrittenen Artikel 301 des türkischen Strafgesetzbuchs (,Verunglimpfung des Türkentums") tatsächlich in einem schlechten Licht erscheinen. Wenig konstruktiv ist allerdings auch der von der französischen Nationalversammlung kürzlich verabschiedete Gesetzentwurf zur Kriminalisierung der Leugnung des türkischen Genozids an den Armeniern. Durch eine Politik der gegenseitigen Beschämung - Stellt die Türkei nun die Leugnung imperialer Gräueltaten in den ehemaligen französischen Kolonien unter Strafe? - wird die Idee einer wechselseitigen Öffnung der nationalen Geschichtsschreibungen geradezu konterkariert. Andererseits sollte es einem Land natürlich nicht verwehrt sein, grenzüberschreitend auf allzu selbstgefällige Geschichtsinterpretationen bis hin zu offensichtlich verdrängten Barbareien kritisch hinzuweisen. Die Bereitschaft zur konstruktiven Auseinandersetzung mit berechtigter Kritik aus einem anderen Mitgliedstaat, der seinerseits gehaltvolle Vorwürfe prüft und gegebe- 
nenfalls sein Geschichtsbild modifiziert, wäre ein weiterer Pfeiler eines im Wesentlichen prozedural verstandenen acquis historique.

Zielpunkt eines derartigen „gemeinsamen historischen Besitzstands" könnte dann möglicherweise ein legitimationsstiftender, geschichtlich fundierter europäischer Verfassungspatriotismus sein, der sich - unabhängig von der Existenz einer expliziten Verfassungsurkunde - weder aus einer euphorischen quasi-bundesstaatlichen ,Meistererzählung speist, noch eine bloße Summe der jetzigen gliedstaatlichen Geschichtsdeutungen darstellt. Ein solcher acquis historique würde sich vielmehr dadurch auszeichnen, dass er in dem Bewusstsein der historischen Exzesse des Nationalismus eine wechselseitige Öffnung der jeweils verzerrten nationalen historischen Identitäten anstrebt, also eine horizontale Europäisierung mitgliedstaatlicher Geschichtsschreibungen.

Teilaspekte einer geschichtsbewussten europäischen Verfassung

Der im Verfassungsvertrag niedergelegte Leitspruch „In Vielfalt geeint“ deutet bereits darauf hin, dass es wohl die besondere Aufgabe der Union ist, historisch gewachsene nationale Politiken und Identitäten ,,autonomieschonend und gemeinschaftsverträglich" (Fritz W. Scharpf) zu koordinieren. Aus diesem Blickwinkel erscheint der immer noch regelmäßig vorgenommene Versuch, die Europäische Union irgendwo auf einem Kontinuum zwischen Bundesstaat und Staatenbund zu verorten, eher nebensächlich oder gar unangebracht. Einige Gründer der Europäischen Gemeinschaften hatten zwar eine dezidiert bundesstaatliche Vision, und im Laufe des Integrationsprozesses hat die Union auch jenseits materiellrechtlicher Kompetenzen immer mehr quasi-staatliche Elemente hinzugewonnen (man denke nur an die Symbole der Union nach Artikel I-8 Verfassungsvertrag - Flagge, Hymne, Leitspruch, Währung, Europatag - und die schon mit dem Maastrichter Vertragswerk geschaffene Uni- onsbürgerschaft). Die Besonderheit der europäischen Politie dürfte aber gerade das Projekt einer föderalen (Rechts-) Gemeinschaft sein, in der nationale Verfassungen durch ein Prinzip „konstitutioneller Toleranz“ (Joseph H. H. Weiler) horizontal miteinander verknüpft sind, anstatt einem starren bundesstaatlichen Vorrangprinzip untergeordnet zu werden.

Vor diesem Hintergrund fußt Christian Joerges' Vorschlag, das supranationale Gemeinschaftsrecht als autonomieschonendes Kollisionsrecht zu konzipieren, auf der Idee, zwei konfligierenden Regulierungen jeweils bestmöglich gerecht zu werden; die eine Rechtsordnung soll nicht einfach der anderen weichen. Dieses Modell kann freilich nicht auf alle Politikfelder gleichermaßen angewendet werden. Besonders sensible Bereiche sollten im Wettbewerb der nationalen Systeme wohl bis zu einem gewissen Maß vor einem ruinösen run to the bottom geschützt werden. Sonst besteht die Gefahr, dass die Mitgliedstaaten auf tiefgreifenden Folgeproblemen des Binnenmarkts sitzen bleiben, die sie allein nicht mehr beheben können, für deren Lösung sich aber auch auf supranationaler Ebene keine ausreichenden Mehrheiten finden. Aus dieser Perspektive sind nationale und europäische output-orientierte Legitimationsprobleme zwei Seiten einer Medaille.

Eine diffuse Angst vor dem Verlust sozialer Errungenschaften brachte zahlreiche Franzosen (neben anderen Beweggründen) dazu, gegen den Verfassungsvertrag zu stimmen. Dies zeigt einmal mehr, dass die Zukunft des Nationalstaats eng mit der des Sozialstaats verzahnt ist. Es ist kein Zufall, dass die europäische Sozialpolitik - abgesehen von der erfolgreichen Antidiskriminierungs- und Gleichstellungspolitik - im Vergleich zu anderen Gemeinschaftspolitiken unterentwickelt ist: Insbesondere redistributive Politik wird vielfach immer noch als Domäne der Nationalstaaten und ihrer, Schicksalsgemeinschaften' gesehen. Die Verbundenheit mit den Unionsbürgern anderer Mitgliedstaaten reicht 
offenbar noch längst nicht so weit, ihnen auch nur einen annährend gleichen Zugang zu den nationalen Sozialsystemen zu gewähren. So werden die mitgliedstaatlichen Kollektive wohl auch in Zukunft im Wesentlichen unter sich umverteilen. Die vage Hoffnung auf einen europäischen ,Dritten Weg' mag als $\mathrm{Zu}$ kunftsvision bestechend sein, kann aber nicht darüber hinwegtäuschen, dass in der Union viele historisch gewachsene, unterschiedliche Sozialstaatssysteme koexistieren. Dieser Komplexität in zunehmend liberalisierten Märkten mit wachsendem Wettbewerbsdruck gerecht $\mathrm{zu}$ werden, ist für das europäische Mehrebenensystem keine leichte Aufgabe, da die für die Lösung größerer Probleme notwendigen Regulierungskompetenzen regelmäßig zwischen Gemeinschaft und Mitgliedstaaten verteilt sind und folglich gemeinsame Strategien erfordern.

Kollektive Identitäten schaffen Solidaritätsressourcen und Freiheit für die Identifizierten. Gleichzeitig ist dem Konstrukt ,Identität ' Abund Ausgrenzung inhärent. Eine europäische Verfassungsidentität, mit der die Hoffnung auf eine stärkere Unterstützung für das Integrationsprojekt verbunden ist, wird sich wohl kaum in strikter Abkehr von den nationalen Identitäten entwickeln lassen. Als überwölbende Gemeinschaftsidee könnte sie ihre Legitimität jedoch gerade daraus gewinnen, dass sie die mitgliedstaatlichen Identitätskonstruktionen grundsätzlich friedlich koexistieren lässt und nur dort in Frage stellt, wo diese gegen das Prinzip der konstitutionellen Toleranz verstoßen. In der Form eines Korrektivs für diskriminierende und ausgrenzende Handlungen von Nationalstaaten wird eine europäische Verfassung auch ihrer historischen Verantwortung gerecht. In seinem Tagungspapier schreibt Konrad H. Jarausch, die ,zentrale Spannung zwischen dem Potenzial zum Töten und Heilen ist das Charakteristikum der faustischen Zivilisation, die von Europa ausging, die Welt unterjochte, den Kontinent fast ruinierte, aber danach dennoch wieder aufrichtete. Nur wenn sie sich diesen Widersprüchen stellt und sie kritisch reflektiert, wird eine neue Verfassung Europas ihre eigene Notwendigkeit historisch überzeugend begründen können“.

\section{Umfassender Überblick zum Thema Föderalismus}

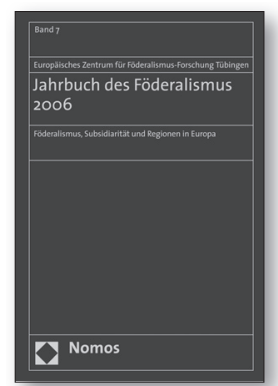

Jahrbuch des Föderalismus 2006

Vom Europäischen Zentrum für Föderalismus-

Forschung Tübingen

2006, 683 S., geb., 76,-€, ISBN 978-3-8329-2147-7

"Das Buch ist ein „Muss" für alle mit dem Föderalismus und der Reform Befassten sowie für alle an den einzel-

nen Themen Interessierten." Erich Röper, Das Parlament 3/06

Bitte bestellen Sie bei Ihrer Buchhandlung oder bei Nomos

C07221/2104-37| -43 | www.nomos.de | sabine.horn@nomos.de

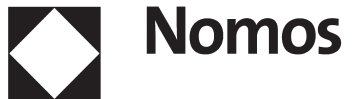

